\title{
FrüHLINGSSPAZIERgäNGe IN SACHSEN
}

\author{
Sabine Webersinke
}

$\mathrm{D}$ er Frühling kommt bestimmt...". So beginnt der Aufruf des Sächsischen Staatsministeriums für Umwelt und Landwirtschaft an alle Interessenten, sich 2016 wieder an den Frühlingsspaziergängen zu beteiligen. Bereits zum 13. Mal lädt das Staatsministerium mit Unterstützung von Sachsenforst und verschiedenen sächsischen Umweltbildungseinrichtungen zu seinem Frühjahrsprogramm ein. Es bietet Verbänden, Vereinen, Kommunen, Verwaltungs- und Umweltbildungseinrichtungen sowie Privatpersonen die Gelegenheit, in ihren Regionen großen und kleinen Entdeckern die Schönheit und Vielfalt der sächsischen Natur nahe zu bringen und somit einen Beitrag zur Bewahrung der Naturschönheiten zu leisten. Der Frühling ist dafür die ideale Jahreszeit. »Die Tage werden wieder länger, der Frühling klopft an die Tür. Mit seinen Sonnenstrahlen erhellt er die Gemüter, verbreitet Freude und macht Lust auf Wandern und Spazierengehen in der Natur ${ }^{1}$, beschreibt der Sächsische Staatsminister für Umwelt und Landwirtschaft Thomas Schmidt diese Jahreszeit. In fünf sächsische Regionen unterteilt, reichen die kostenfreien Angebote von thematischen Wanderungen über den Besuch von regionalen Sehenswürdigkeiten hin zu botanischen, ornithologischen und ökofaunistischen Führungen in Landschaften, Wäldern, Gärten und Parks.

Seit 2014 beteiligt sich die Arbeitsgruppe Sächsische Gartengeschichte ${ }^{2}$ an den Frühlingsspaziergängen, bieten diese doch eine gute Möglichkeit, auf noch wenig bekannte historische Gartenanlagen aufmerksam zu machen. Die Arbeitsgruppe hat sich die denkmalkundliche Erschließung historischer Gärten in Sachsen zum Ziel gesetzt und veranstaltete 2014 und 2015 jeweils eine Besichtigung eines historischen Gartens oder Parks in der Region Elbland/Sächsische Schweiz.

Am Sonnabend, dem 26. April 2014, fand bei strahlendem Sonnenschein der erste Spaziergang der Arbeitsgruppe durch den Röhrsdorfer Grund ${ }^{3}$ bei Dohna statt. Anja Eppert, freie Landschaftsarchitektin, führte gemeinsam mit dem Heimatverein Röhrsdorf durch die kaum bekannte Anlage, die als eine der ältesten landschaftlichen Parkanlagen Sachsens gilt. Abseits der vielbesuchten Garten- und Parkanlagen, die das Elbtal zu bieten hat, liegt inmitten dicht bewaldeter Hänge dieses Kleinod sächsischer Gartengeschichte. Das Tal der Freundschaft wurde es einmal genannt. Steinerne Zeugen künden bis heute von den innigen Freundschaften der Familie von Carlowitz, die das Gelände seit Ende des 18. Jahrhunderts in ein Idyll der besonderen Art verwandelte. Etwa 45 Erwachsene, acht Kinder und ein Hund begaben sich auf die Suche nach gartenkünstlerischen Spuren im Röhrsdorfer Grund und konnten dabei die bisher durch den Heimatverein Röhrsdorf realisierten Wiederherstellungsarbeiten bestaunen. Viele der aus der Nähe stammenden Besucher kannten den Röhrsdorfer Grund und seine Geschichte kaum und waren von der Lieblichkeit der Anlage, aber auch vom Engagement des Heimatvereins beeindruckt.

Der Erfolg des ersten Spazierganges ermutigte die Arbeitsgruppe, sich auch 2015 wieder bei den Frühlingsspaziergängen zu 


\section{FrüHLINGSSPAZIERGÄNGE IN SACHSEN}

beteiligen. Diesmal wurde die Parkanlage am Schloss Thürmsdorf bei Struppen ausgewählt. Für die Führung am 23. Mai 2015 konnte der Gartenbesitzer Sven-Eric Hitzer gewonnen werden, der die Besucher mit seiner Begeisterung und seinen Visionen für die Anlage in den Bann zog. 25 Erwachsene, vier Kinder und zwei Hunde spazierten zunächst auf dem Malerweg Sächsische Schweiz, der am Thürmsdorfer Park entlang führt. Am Thürmsdorfer Mausoleum öffnete sich eine grandiose Aussicht auf die Festung Königstein. Diese Aussicht war vor 200 Jahren ein beliebtes Motiv für Maler wie beispielsweise den kursächsischen Hofmaler Johann Alexander Thiele und gehört heute zu den fast in Vergessenheit geratenen Ausblicken. An Feldern entlang ging es über eine Streuobstwiese in den landschaftlich gestalteten Park, den der königlich-sächsi- sche Gartenbaudirektor Max Bertram 1912 im Auftrag des damaligen Besitzers, Freiherrn von Biedermann, schuf. Die Anlage zählt zum Spätwerk Bertrams und stellt die Krönung seines Schaffens dar. Die Besucher waren nicht nur von der landschaftlich schönen Lage, sondern auch von der Größe und Schönheit des doch kaum bekannten Gartens beeindruckt. Im Anschluss bestand die Möglichkeit, Köstlichkeiten der benachbarten Schokoladenmanufaktur Adoratio kennen zu lernen.

Die Arbeitsgruppe Sächsische Gartengeschichte plant auch für 2016 einen Spaziergang durch eine kaum bekannte Gartenanlage in Sachsen. Ab Januar 2016 wird das Programm der Frühlingsspaziergänge auf www.natur.sachsen.de zu finden sein.
1 Schmidt, Thomas: Einleitung, in: Sächsisches Staatsministerium für Umwelt und Landwirtschaft (Hg.): Programmheft Frühlingsspaziergänge 2015, Dresden 2015, S. 2.

2 Der AG Sächsiche Gartengeschichte gehören an: Silke Epple, Nora Kindermann, Marcus Köhler, Stefanie Krihning, Erika Schmidt, Henrike Schwarz, Sabine Webersinke.
3 Eppert, Anja: Der Röhrsdorfer Grund. Auf den Spuren einer der ältesten landschaftlichen Anlagen Sachsens/ enthält: Georg Engert: Der Röhrsdorfer Grund. Dresden (1912), Mitteilungsheft der Pückler-Gesellschaft e.V. Berlin, N.F. Heft 27, Berlin 2013..

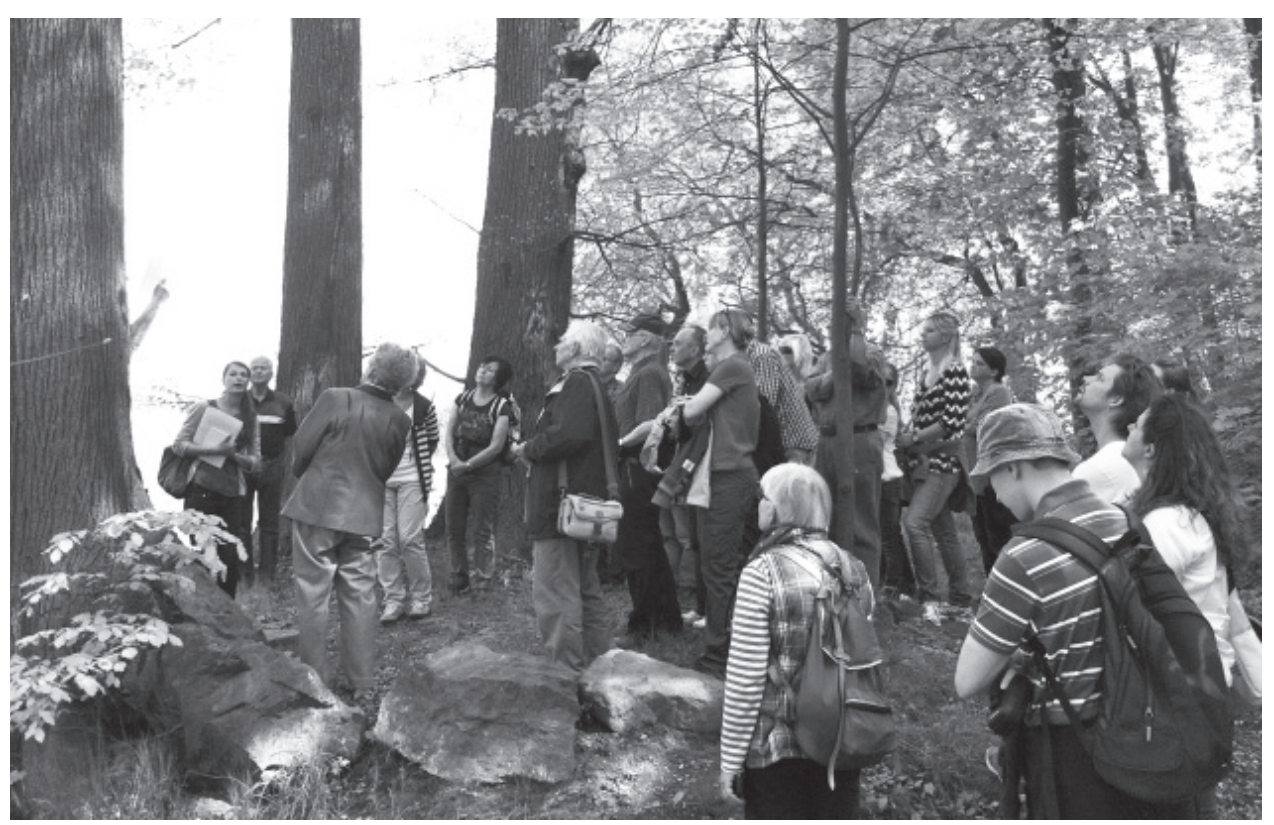

1 | Frühlingsspaziergang mit Anja Eppert durch den Röhrsdorfer Grund bei Dohna, April 2014 (Fotografie, Sabine Webersinke, 2014). 